\title{
Dietary Flax Oil During Pregnancy and Lactation Retards Disease Progression in Rat Offspring With Inherited Kidney Disease
}

\author{
DEEPA SANKARAN, NEDA BANKOVIC-CALIC, CLAUDIA YU-CHEN PENG, MALCOLM R. OGBORN, \\ AND HAROLD M. AUKEMA
}

Department of Human Nutritional Sciences [D.S., Y.-C.P., M.R.O., H.M.A.], Department of Pediatrics and Child Health [N.B.-C., M.R.O.], University of Manitoba, Winnipeg, Manitoba R3T 2N2, Canada; Manitoba Institute of Child Health [M.R.O., H.M.A.], Winnipeg, Manitoba R3E 3P4, Canada

\begin{abstract}
Dietary flax oil (FO) retards disease progression in growing or adult animal models of kidney disease. To determine whether dietary flax oil during the perinatal period would alter renal disease progression in offspring, Han-SPRD-cy rats with inherited cystic kidney disease were given diets with either $7 \%$ FO or corn oil (CO), throughout pregnancy and lactation. At 3 wk of age, offspring were then given either the same or the alternate diet for $7 \mathrm{wk}$. Rats given FO during the maternal period had $15 \%$ less renal cyst growth compared with rats given FO only in the postweaning period. Dietary FO, compared with $\mathrm{CO}$, in the maternal period also resulted in $12 \%$ lower cell proliferation and $15 \%$ less oxidant injury in diseased kidneys of offspring. Including FO in both the maternal and postweaning period resulted in 29-34\% less renal interstitial fibrosis and 22-23\% lower glomerular hypertrophy. Along with improved histology, these rats exhibited $13 \%$ less proteinuria and $30 \%$ lower creatinine clearance when dietary FO was given in the maternal period. The potential for dietary FO during pregnancy and lactation to positively modulate adult renal disease has significant implications for the 1 in 1000 individuals with congenital cystic kidney disease.
\end{abstract} (Pediatr Res 60: 729-733, 2006)

$\mathrm{C}$ Thronic kidney disease is a gradual irreversible loss of kidney function which usually leads to end-stage renal disease and the need for expensive renal replacement therapies. Its increasing prevalence ( $>50 \%$ rise in $10 \mathrm{y}$ in the U.S and a $19 \%$ rise in $5 \mathrm{y}$ in Canada) $(1,2)$ and staggering healthcare costs warrant further inquiry into treatment options that slow or prevent its progression. Dietary interventions such as altering dietary fat sources slow the progression of chronic renal disease. Dietary n-3 fatty acids from fish and flax sources have been shown to be reno-protective, particularly in renal injury that has an immune and/or inflammatory component (3-7). These protective effects of n-3 fatty acids on renal disease progression in humans or animal models have been demonstrated in adult or weanling animals.

Received February 27, 2006; accepted July 8, 2006.

Correspondence: Harold Aukema, Ph.D., University of Manitoba, H506 Duff Roblin, Winnipeg MB, Canada R3T2N2; e-mail: aukema@umanitoba.ca

Supported by grants from National Science and Engineering Research Council of Canada and Manitoba Institute of Child Health, Manitoba, Canada and a Manitoba Health Research Council Graduate Fellowship (DS).

DOI: 10.1203/01.pdr.0000245912.78078.d5
The fetal programming hypothesis suggests that the first environment, the intrauterine, also influences the risk of chronic diseases in adult life (8). For example, higher maternal saturated fat intake has been linked to hyperinsulinemic, hyperglycemic, pro-atherogenic and hypertensive phenotypes in offspring (9-11), while a study with high maternal n-3 fatty acids intake failed to show such deleterious fetal programming effects (12). Impaired fetal nephrogenesis and reduced nephron number can result from maternal malnutrition and are associated with adult hypertension in humans (13) as well as experimental models of developmental programming (1416). These reports indicate that renal organogenesis is susceptible to changes in the fetal environment which can modulate development of chronic diseases later in life.

The Han:SPRD-cy rat is an established model of autosomal dominant polycystic kidney disease (PKD) in which heterozygotes develop progressive cystic expansion, renal interstitial fibrosis, inflammation and oxidative damage, as well as hypertension and uremia in adult life (17). Dietary flax (rich in n-3 fatty acids) reduces renal interstitial fibrosis, cystic growth and renal macrophage infiltration in this model of hereditary kidney disease when given in the postweaning period of life $(7,18)$. The aim of the current study was to determine whether flax oil supplementation during pregnancy and lactation would slow the progression of renal disease in offspring of Han: SPRD-cy rats. To further delineate the effects of maternal dietary flax oil supplementation on renal injury, a group of animals that were given flax oil during both the maternal and postweaning periods as well as a group of animals that were fed flax oil only in the postweaning period were included.

\section{METHODS}

Animals. All animal procedures were approved by the University of Manitoba Committee on Animal Care and were in accordance with the guidelines of the Canadian Council on Animal Care. Breeder Han:SPRD-cy

Abbreviations: CO, corn oil; FO, flax oil; PCNA, proliferating cell nuclear antigen; PKD, polycystic kidney disease 
rats (also known as $\mathrm{PKD} / \mathrm{Mhm}(c y /+)$ rats) were derived from our breeding colony, which originated from the colony of Dr. Benjamin Cowley, University of Kansas Medical Center, Kansas City (17). They were randomly assigned to either a flax oil diet (FO, 7\% oil, rich in 18:3n-3) or a corn oil control diet (CO, 7\% oil, rich in linoleic acid, 18:2n-6) two weeks before mating and throughout pregnancy and lactation. Male offspring from both groups were weaned at three weeks of age and were given either the same maternal diet or the alternate diet for another seven weeks. Diet ingredients, with the exception of flax oil, were purchased from Dyets Inc. (Bethlehem, PA) or Harlan Teklad (Madison, WI). Flax oil (FO) was a generous gift from Bioriginal Food and Science Corporation (Saskatoon, SK). Diets were based on the AIN93G guidelines for rodent diets for optimal growth and adequate nutrition $(19,20)$. Hence the diets were identical (including calorie, carbohydrate, protein, mineral and vitamin content) except for the fat source.

Rats were weighed weekly and placed in metabolic cages for urine collection a week before termination. At the end of the feeding period, rats were anesthetized with $\mathrm{CO}_{2}$ overexposure, decapitated and trunk blood was collected to obtain serum. Left kidneys were removed and weighed. The left kidney was sectioned in half longitudinally across the hilum, and one-half of wet kidney tissue was fixed in $10 \%$ buffered formalin for morphologic and histologic analyses.

Renal biochemistry. Serum urea was determined spectrophotometrically using reagents from Sigma Chemical Co. kit 640-A (Sigma Chemical Co., MO). Serum creatinine and urinary creatinine were measured using a method developed by Heingard and Tiderstrom and adapted for a micro-assay procedure using 96-well plates. Urinary protein was measured using the Bradford method for total protein.

Histology and immunohistochemistry. The formalin-fixed left kidney was embedded in paraffin, sectioned at $5 \mu \mathrm{m}$ and was processed using our previously described methods for histologic and immunohistochemical analyses $(7,19)$. Transverse tissue sections (including cortex, medulla and papilla) were stained with hematoxylin and eosin for cyst area measurement and those for quantitative analysis of fibrosis with Sirius red (in adaptation of Masson's trichrome stain) which permits image analysis measurement using a standard incandescent microscope light source. Renal cell proliferation was assessed using a 1:50 dilution of an anti-mouse proliferating cell nuclear antigen (PCNA) antibody (M0879, Dako Corporation, Carpinteria, CA). Renal inflammation and oxidant injury were detected using 1:50 dilutions of a mouse anti-rat monocytes/macrophages MAb (MAB 1435, Chemicon International, Temecula, CA) and a rabbit anti-Cu 2+-oxidized LDL polyclonal antibody (AB3230, Chemicon International, Temecula, CA), respectively. The Dako EnVision Plus system (K4008, Dako Corporation, Carpinteria, CA) was used for secondary detection. Animals were classified as affected by an experienced, blinded observer (NBC) on the basis of cyst growth and pathology that is characteristic of the disease.

Image analysis. After being captured using a SPOT junior CCD camera by random stage movement through the sections, images were analyzed using the Image Pro version 4.5 package (Media Cybernetics, Silver Spring, MD) as described previously $(7,19)$. An average of 25 measurements starting from a random field of tissue section from kidney cross-sections were collected for all histomorphometric analyses. All measurements were carried out in a blinded fashion. The portion of tissue section occupied by tubular lumen or cyst was determined for cyst area measurements. Renal interstitial fibrosis and oxidant injury were measured by densitometry, while the number of cells stained positive for PCNA as well as macrophages were counted using the Image Pro version 4.5 package (Media Cybernetics, Silver Spring, MD) as described previously $(7,19)$. Renal cyst area measurements were expressed relative to kidney weight-to-body weight ratios. PCNA positive cells in tubular epithelial cells were counted as these are the base cell type of cysts. Measurements of fibrosis, oxidized LDL and other cellular markers were corrected to solid tissue areas of sections to avoid underestimation of these variables due to empty cystic areas in these sections.

Using standard sterological techniques developed by Weibel (21), mean glomerular volume (MGV) was determined by measuring glomerular diameters of 30 randomly chosen glomeruli per kidney by light microscopy using hematoxylin and eosin stained sections. Using the aforementioned imaging system and a $20 \times$ calibration grid, the maximum diameter of each glomerulus was measured and converted to an estimate of glomerular volume using the following formula: mean glomerular area (MGA) was calculated as MGA $=$ $\pi \mathrm{r}^{2}$. MGV was calculated as $\mathrm{MGV}=1.25(\mathrm{MGA})^{3 / 2}$. The value of 1.25 is derived from $\beta / \mathrm{K}$, which are coefficients based on assumptions made for the maximum diameter of spheres $(\beta=1.38)$ and the distribution bias of section location $(\mathrm{K}=1.10)(22)$.

Statistical analyses. Data were analyzed using a general linear model ANOVA followed by post hoc $t$-tests when interactions were present, using SAS software (SAS, Cary, NC). Normality of the data were tested using a plot of actual versus predicted residuals and the Shapiro-Wilk's W statistic on the residuals. Data were normalized by logarithmic transformation if necessary. Post hoc $t$-tests were performed only if the main or interaction effects were significant at $p<0.05$ or $p<0.1$, respectively.

\section{RESULTS}

During the study period, all animals thrived on the diets. The diets given during the maternal period did not affect either body weights at weaning (pups given $\mathrm{CO}=55.5 \mathrm{~g} \pm 1.1 \mathrm{~g}$ and pups given $\mathrm{FO}=56.7 \mathrm{~g} \pm 1.1 \mathrm{~g}$ ) or litter size (mean litter size $=$ $8.3 \pm 0.7$ for female breeders given $\mathrm{CO}$ and $\mathrm{FO}$ ).

Dietary FO compared with $\mathrm{CO}$ in the maternal period improved renal histology and function in diseased offspring. The primary defect in Han:SPRD-cy rats is the growth and development of renal cysts that disrupt normal renal architecture, eventually causing structural damage leading to pathology. Dietary FO in the maternal period, alone or in combination with FO in the postweaning period, resulted in 15-28\% less in cyst growth when compared with rats given FO only in the postweaning period (Fig. 1). Less cyst growth resulted in smaller kidneys in these rats, whether expressed as unadjusted weights, relative to body weight (Table 1) or relative to liver weight (data not shown), thus supporting the cyst expansion data. This data indicates that the dietary FO in the maternal period had disease mitigating effects on the primary defect in this model of kidney disease.

Dietary FO in the maternal period also resulted in $14 \%$ fewer PCNA positive cells, reflecting less cell proliferation (Fig. 2). This protective effect of dietary FO in the maternal period was as effective as dietary FO in the postweaning period. When FO was given in both time periods, no further benefit on cell proliferation was observed. A similar pattern was observed for oxidant injury (Fig. 3). Dietary FO in the maternal and postweaning periods independently resulted in less staining for oxidized LDL (33\% and $45 \%$ less, respectively, compared with rats given $\mathrm{CO}$ in both feeding periods). Combining the dietary FO treatments in both feeding periods did not enhance the effect of FO in the postweaning period alone. Renal inflammation was not significantly altered by dietary FO in the maternal period, but was $21 \%$ lower with FO in the postweaning period (Fig. 4).

With respect to renal function, diseased rats compared with normal rats had worsened renal function as indicated by blood

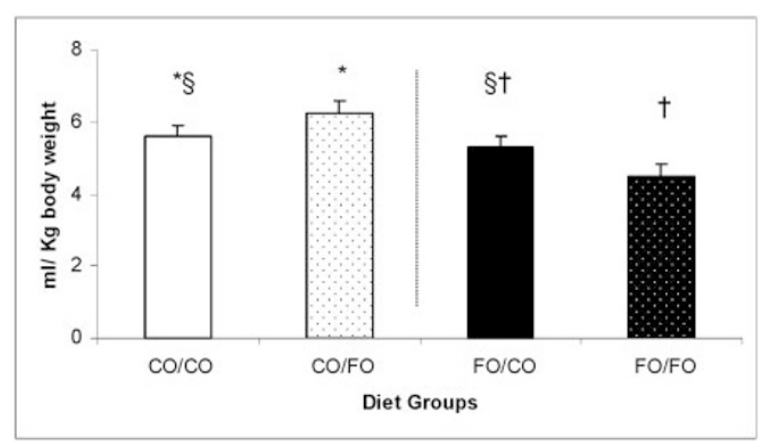

Figure 1. Renal cystic change in Han:SPRD-cy rats given dietary FO compared with $\mathrm{CO}$ diets in the maternal and/or postweaning periods. Groups are denoted as maternal period/postweaning period $(n=14-18)$. Values are means \pm SEM. Maternal $\times$ postweaning period interaction $(p=0.0255)$. Means with different symbols denote significant differences $(p<0.05)$. 
Table 1. Kidney weights, serum and urine biochemistry in diseased (cy/+) and normal (+/+) Han:SPRD-cy rats given dietary FO and $\mathrm{CO}$ in the maternal and/or post-weaning periods

\begin{tabular}{|c|c|c|c|c|c|}
\hline Maternal/postweaning period & $\mathrm{CO} / \mathrm{CO}$ & $\mathrm{CO} / \mathrm{FO}$ & $\mathrm{FO} / \mathrm{CO}$ & $\mathrm{FO} / \mathrm{FO}$ & Significant effects $p$ \\
\hline$c y /+$ Rats & $\mathrm{N}=15$ & $\mathrm{~N}=18$ & $\mathrm{~N}=19$ & $\mathrm{~N}=19$ & \\
\hline Body weight $(\mathrm{g}) \S$ & $333 \pm 5.6 \pi$ & $328 \pm 5.14$ & $332 \pm 5.14$ & $306 \pm 4.8 \dagger$ & Interaction $(P=0.0515)$ \\
\hline Kidney weight (g/100g body weight) & $2.44 \pm 0.1 \Phi$ & $2.62 \pm 0.1^{*}$ & $2.29 \pm 0.1 \Phi$ & $2.00 \pm 0.1 \dagger$ & Interaction $(P=0.0104)$ \\
\hline Serum creatinine $(\mu \mathrm{mol} / \mathrm{L})$ & $76.4 \pm 4.3$ & $67.6 \pm 3.8$ & $81.7 \pm 3.6$ & $79.3 \pm 3.7$ & Maternal period $(P=0.0307)$ \\
\hline Serum urea $(\mathrm{mmol} / \mathrm{L})$ & $33.9 \pm 2.2$ & $28.9 \pm 2.0$ & $36.8 \pm 1.9$ & $38.1 \pm 1.9$ & Maternal period $(P=0.0039)$ \\
\hline Creatinine clearance $(\mathrm{mL} / \mathrm{min} / 100 \mathrm{~g}$ body weight $)$ & $0.22 \pm 0.02$ & $0.27 \pm 0.02$ & $0.18 \pm 0.02$ & $0.17 \pm 0.02$ & Maternal period $(P=0.0031)$ \\
\hline Body weight $(\mathrm{g})$ & $350 \pm 6.0$ & $342 \pm 7.9$ & $357 \pm 9.1$ & $355 \pm 7.9$ & None \\
\hline Kidney weight (g/100g body weight) & $0.76 \pm 0.02$ & $0.73 \pm 0.03$ & $0.73 \pm 0.03$ & $0.69 \pm 0.03$ & None \\
\hline Kidney weight $(\mathrm{g})$ & $2.58 \pm 0.1$ & $2.50 \pm 0.1$ & $2.63 \pm 0.1$ & $2.47 \pm 0.1$ & None \\
\hline Serum creatinine $(\mu \mathrm{mol} / \mathrm{L})$ & $42.2 \pm 1.7$ & $37.0 \pm 2.2$ & $39.2 \pm 2.3$ & $39.5 \pm 2.3$ & None \\
\hline Serum urea $(\mathrm{mmol} / \mathrm{L})$ & $8.7 \pm 0.5$ & $9.0 \pm 0.7$ & $7.2 \pm 0.8$ & $9.0 \pm 0.8$ & None \\
\hline Creatinine clearance $(\mathrm{mL} / \mathrm{min} / 100 \mathrm{~g}$ body weight $)$ & $0.44 \pm 0.02$ & $0.48 \pm 0.03$ & $0.49 \pm 0.02$ & $0.45 \pm 0.02$ & None \\
\hline
\end{tabular}

$\S$ Data are expressed as least squares means. Means within a row with different symbols are significantly different $(P<0.05)$.

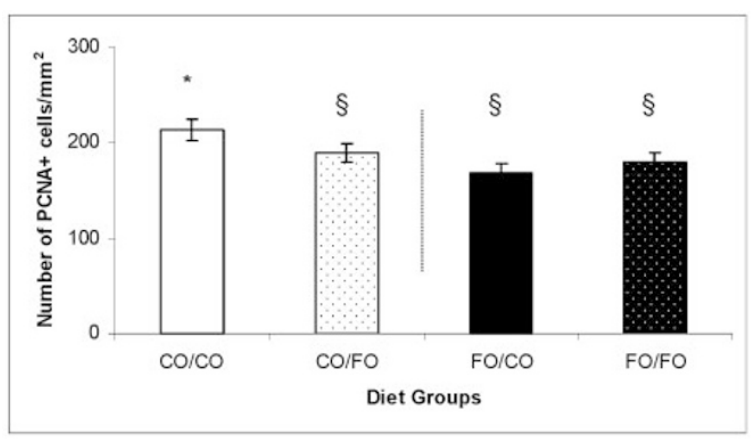

Figure 2. Renal cells positive for PCNA in Han:SPRD-cy rats given dietary FO compared with $\mathrm{CO}$ in the maternal and/or postweaning periods. Groups are denoted as maternal period/postweaning period $(n=14-18)$. Values are means \pm SEM. Maternal $\times$ postweaning period interaction $(p=0.0291)$. Means with different symbols denote significant differences $(p<0.05)$

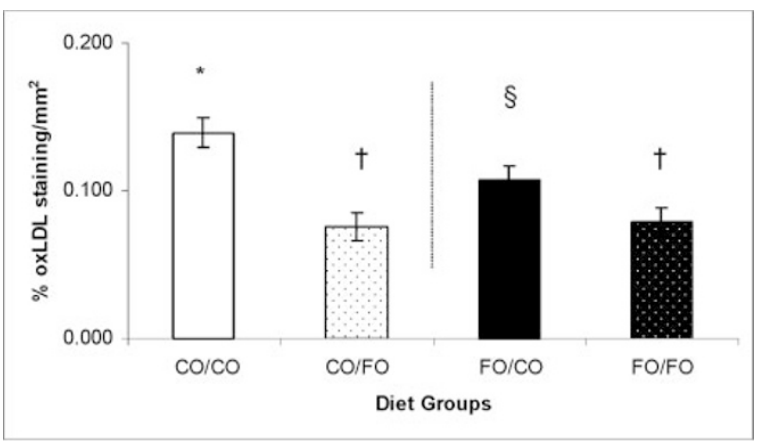

Figure 3. Renal oxidized-LDL staining in Han:SPRD-cy rats given dietary FO compared with $\mathrm{CO}$ in the maternal and/or postweaning periods. Groups are denoted as maternal period/postweaning period $(n=14-18)$. Values are means \pm SEM. Maternal $\times$ postweaning period interaction $(p=0.0816)$. Means with different symbols denote significant differences $(p<0.05)$.

and urine parameters (Table 1). Concurrent with its beneficial effects on histology, dietary FO given during the maternal period resulted in less proteinuria $(p=0.0125)$ and lower creatinine clearance $(p=0.0001)$. This reduction in creatinine clearance was associated with higher levels of serum urea $(p=0.0039)$ and creatinine $(p=0.0307)$

To further probe glomerular and interstitial changes associated with reduced proteinuria in rats given FO during the

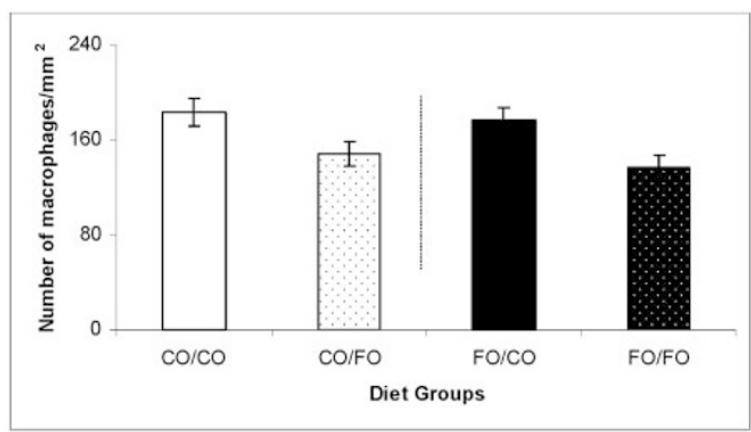

Figure 4. Renal macrophage count in Han:SPRD-cy rats given dietary FO compared with $\mathrm{CO}$ in the maternal and/or postweaning periods. Groups are denoted as maternal period/postweaning period $(n=14-18)$. Values are means \pm SEM. Postweaning period $(p=0.0007$, FO $<\mathrm{CO})$.

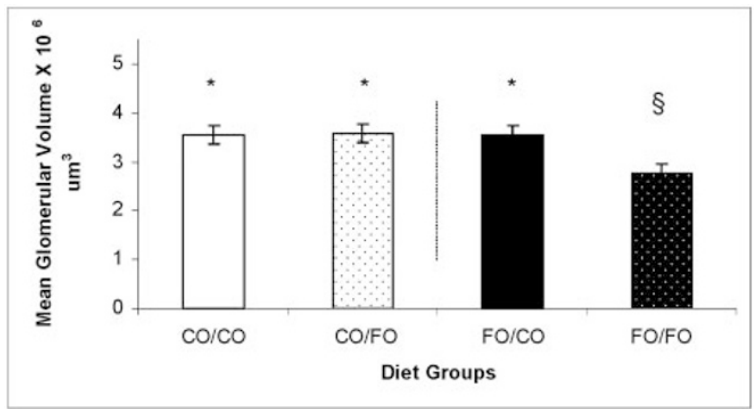

Figure 5. Mean glomerular volume in Han:SPRD-cy rats given dietary FO compared with $\mathrm{CO}$ in the maternal and/or postweaning periods. Groups are denoted as maternal period/postweaning period $(n=14-18)$. Values are means \pm SEM. Maternal $\times$ postweaning period interaction $p=0.0160$ ). Means with different symbols denote significant differences $(p<0.05)$.

maternal period, an examination of mean glomerular volumes and renal interstitial fibrosis was undertaken. Glomerular hypertrophy (Fig. 5) and renal interstitial fibrosis (Fig. 6) and were altered by dietary $\mathrm{FO}$ when given in the maternal period. However, to manifest this effect, dietary FO also had to be included in the postweaning period. When dietary FO was given in both feeding periods, glomerular hypertrophy was lower by $22-23 \%$ (Fig. 5) and interstitial fibrosis was $19-30 \%$ 


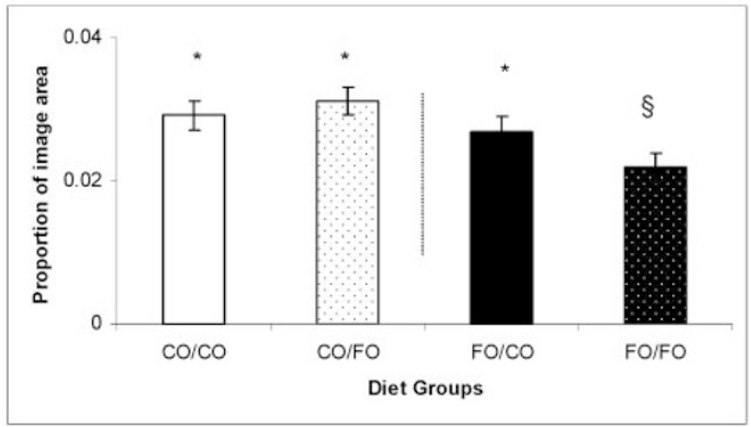

Figure 6. Renal interstitial fibrosis in Han:SPRD-cy rats given dietary FO compared with $\mathrm{CO}$ in the maternal and/or postweaning periods. Groups are denoted as maternal period/postweaning period $(n=14-18)$. Values are means \pm SEM. Maternal $\times$ postweaning period interaction $(p=0.0378)$. Means with different symbols denote significant differences $(p<0.05)$.

less (Fig. 6), compared with any treatment that included dietary $\mathrm{CO}$ in either feeding period.

\section{DISCUSSION}

This study demonstrates that dietary supplementation with FO initiated during pregnancy and lactation reduces histologic renal injury in offspring of Han:SPRD-cy rats. Dietary FO given in the maternal period alleviated increases in cyst expansion, kidney size, renal cell proliferation, oxidant damage, interstitial fibrosis, glomerular hypertrophy and proteinuria. The concept that the events encountered in fetal life can have life-long consequences and affect adult health is rapidly gaining acceptance. There is growing evidence that maternal nutritional status can alter the state of the fetal genome and program gene expression in normal embryonic kidney development (16). The current study further extends the above observations that maternal nutrition also can have long term effects on the postnatal kidney afflicted by a hereditary disease. Autosomal dominant PKD is a developmental disorder, affecting 1:400 to 1:1000 people and, is the most common genetic cause of renal failure in humans (23). Localization of the PKD1 and PKD2 genes has made it possible to use genetic markers to determine the status of asymptomatic individuals from families with a history of PKD, even as early as prenatally (23). This ability for early identification of PKD allows for subsequent preventative therapy such as dietary intervention during the maternal period as demonstrated in the current study.

The beneficial effects of flax oil given maternally are consistent with previous studies that reported amelioration of renal injury when flax oil intervention was introduced in the adult or postweaning stages $(6,7,19)$. In cystic kidney diseases, recent evidence suggests that cyst expansion precedes the changes in function and is associated with the initiation of inflammation, fibrosis and other secondary changes (24). Thus, therapeutic interventions that reduce the rate of cystic growth will ameliorate development of late-onset renal insufficiency (24). Dietary FO in the maternal period not only reduced cyst expansion and kidney size in diseased rat offspring but also resulted in improvements in other histologic parameters and function (proteinuria). Proteinuria is not only a marker of compromised kidney function, but it also is a key indicator of prognosis and therapy of chronic renal injury (25).

Although the reduction in creatinine clearance may appear to be contradictory to the observed benefits on cyst expansion and renal histology, a decrease in GFR is not always associated with worsening of disease. In the Modification of Diet in Renal Disease study, low protein diets had seemingly contrasting short- and long-term effects on GFR. In this study, adults with moderate renal injury in the low compared with normal protein diet group had an initial faster mean decline in GFR but a slower mean decline in GFR thereafter (26). This initial decrease in GFR followed by long-term reduction in disease progression by dietary protein restriction also has been demonstrated in an animal model (27). Thus, it is possible that an initial short-term reduction in creatinine clearance with dietary FO in the maternal period is followed by a slower decline in GFR in the long term. We speculate that resistance to glomerular hypertrophy is linked to the reduced proteinuria we have observed; in turn the lower proteinuria may explain the reduced interstitial fibrosis we have demonstrated in animals given dietary FO. A longer-term study that examines the effects of dietary FO in the maternal period on GFR and the ultimate outcome of renal disease is needed to definitively address this issue.

Glomerular hypertrophy and other glomerular abnormalities have been reported in human PKD kidneys (28) as well as in 6-mo-old cystic rat kidneys (29). The glomerulus is often ignored in PKD, probably because the tubular cystic changes are more dramatic. However, although glomerular changes occur later in the disease process, these changes are important since it is the failure of glomerular filtration that eventually leads to end stage renal failure. The current study is the first to demonstrate a dietary effect on mean glomerular volume in cystic kidney disease, demonstrating the potential for dietary interventions during the maternal period to counter the glomerular abnormalities in this disorder.

Dietary supplementation of flaxseed or FO in rat dams increases 18:3n-3 ( $\alpha$-linolenic acid) and reduces 20:4n-6 (arachidonic acid) in the milk as well as in the tissues of offspring $(30,31)$ confirming that there is both placental and milk transfer of these fatty acids. The high $\alpha$-linolenic acid content in FO may contribute to the reno-protective effects observed in the current study. FO compared with $\mathrm{CO}$ supplementation in Han:SPRD-cy rat and pcy mouse models of cystic kidney disease leads to an enrichment of renal $\alpha$-linolenic acid and 20:5n-3 (eicosapentaenoic acid), and a reduction of linoleic acid (18:2n-6) and arachidonic acid $(7,19)$. These studies demonstrate that $\alpha$-linolenic acid is converted to longer chain n-3 fatty acids, and inhibits conversion of linoleic acid to arachidonic acid. Since arachidonic acid and eicosapentaenoic acid compete for the same metabolic enzymes, supplementing the diet with n-3 fatty acids may cause a shift in eicosanoid production, with eicosapentaenoic acid competitively inhibiting the metabolism of arachidonic acid to the more detrimental 2 series eicosanoids (32). Not only is the rate of conversion of eicosapentaenoic acid to thromboxane $\mathrm{A}_{3}$ lower than that of arachidonic acid to thromboxane $A_{2}$ (33), but the vasoconstrictor potency of thromboxane $A_{3}$ is also significantly lower 
than that of its 2 series counterpart (34). N-3 fatty acid enrichment with FO also has been associated with antiproliferative effects in renal injury in weanling Han:SPRD-cy rats (7) as well as in cancer models (35-37). These reported anti-proliferative effects are similar to those observed in the current study, suggesting that altered eicosanoid production during the maternal period could attenuate the increased cell proliferation observed in diseased kidneys.

Autosomal dominant PKD is a slowly progressing chronic kidney disease that begins in utero (23). The results from the present study demonstrate that initiating dietary interventions in utero can reduce the progression of this genetically determined kidney disease. The findings may be relevant to other juvenile genetic/inherited cystic renal diseases such as autosomal recessive PKD and juvenile nephronophthisis as well as other renal disorders with a genetic basis such as IgA nephropathy, Alport syndrome and congenital nephrotic syndrome.

Acknowledgements. We would like to thank Kari Hamblin, Ling Lee, Laurie Evans and Leah Cahill for their technical assistance.

\section{REFERENCES}

1. Coresh J, Byrd-Holt D, Astor BC, Briggs JP, Eggers PW, Lacher DA, Hostetter TH 2005 Chronic kidney disease awareness, prevalence, and trends among U.S. adults, 1999 to 2000. J Am Soc Nephrol 16:180-188

2. Chauhan T 2004 End-stage renal disease patients up nearly 19\%. CMAJ 170:1087

3. Donadio JV 2001 The emerging role of omega-3 polyunsaturated fatty acids in the management of patients with IgA nephropathy. J Ren Nutr 11:122-128

4. Lu J, Bankovic-Calic N, Ogborn M, Saboorian MH, Aukema HM 2003 Detrimental effects of a high fat diet in early renal injury are ameliorated by fish oil in Han:SPRD-cy rats. J Nutr 133:180-186

5. Hall AV, Parbtani A, Clark WF, Spanner E, Keeney M, Chin-Yee I, Philbrick DJ, Holub BJ 1993 Abrogation of MRL/lpr lupus nephritis by dietary flaxseed. Am J Kidney Dis 22:326-332

6. Ingram AJ, Parbtani A, Clark WF, Spanner E, Huff MW, Philbrick DJ, Holub BJ 1995 Effects of flaxseed and flax oil diets in a rat-5/6 renal ablation model. Am J Kidney Dis 25:320-329

7. Ogborn MR, Nitschmann E, Bankovic-Calic N, Weiler HA, Aukema H 2002 Dietary flax oil reduces renal injury, oxidized LDL content, and tissue n-6/n-3 FA ratio in experimental polycystic kidney disease. Lipids 37:1059-1065

8. Barker DJ 1995 The fetal and infant origins of disease. Eur J Clin Invest 25:457-463

9. Taylor PD, McConnell J, Khan IY, Holemans K, Lawrence KM, Asare-Anane H, Persaud SJ, Jones PM, Petrie L, Hanson MA, Poston L 2005 Impaired glucose homeostasis and mitochondrial abnormalities in offspring of rats fed a fat-rich diet in pregnancy. Am J Physiol Regul Integr Comp Physiol 288:R134-R139

10. Khan IY, Dekou V, Douglas G, Jensen R, Hanson MA, Poston L, Taylor PD 2005 A high-fat diet during rat pregnancy or suckling induces cardiovascular dysfunction in adult offspring. Am J Physiol Regul Integr Comp Physiol 288:R127-R133

11. Palinski W, D’Armiento FP, Witztum JL, de Nigris F, Casanada F, Condorelli M, Silvestre M, Napoli C 2001 Maternal hypercholesterolemia and treatment during pregnancy influence the long-term progression of atherosclerosis in offspring of rabbits. Circ Res 89:991-996

12. Siemelink M, Verhoef A, Dormans JA, Span PN, Piersma AH 2002 Dietary fatty acid composition during pregnancy and lactation in the rat programs growth and glucose metabolism in the offspring. Diabetologia 45:1397-1403
13. Keller G, Zimmer G, Mall G, Ritz E, Amann K 2003 Nephron number in patients with primary hypertension. N Engl J Med 348:101-108

14. Woods LL, Weeks DA, Rasch R 2004 Programming of adult blood pressure by maternal protein restriction: role of nephrogenesis. Kidney Int 65:1339-1348

15. Langley-Evans SC, Langley-Evans AJ, Marchand MC 2003 Nutritional programming of blood pressure and renal morphology. Arch Physiol Biochem 111:8-16

16. Welham SJ, Riley PR, Wade A, Hubank M, Woolf AS 2005 Maternal diet programs embryonic kidney gene expression. Physiol Genomics 22:48-56

17. Cowley BD Jr, Gudapaty S, Kraybill AL, Barash BD, Harding MA, Calvet JP, Gattone VH 2nd 1993 Autosomal-dominant polycystic kidney disease in the rat. Kidney Int 43:522-534

18. Ogborn MR, Nitschmann E, Weiler H, Leswick D, Bankovic-Calic N 1999 Flaxseed ameliorates interstitial nephritis in rat polycystic kidney disease. Kidney Int 55:417423

19. Sankaran D, Lu J, Bankovic-Calic N, Ogborn MR, Aukema HM 2004 Modulation of renal injury in pcy mice by dietary fat containing n-3 fatty acids depends on the level and type of fat. Lipids 39:207-214

20. Reeves PG, Nielsen FH, Fahey GC Jr 1993 AIN-93 purified diets for laboratory rodents: final report of the American Institute of Nutrition ad hoc writing committee on the reformulation of the AIN-76A rodent diet. J Nutr 123:1939-1951

21. Weibel E 1979 Practical methods for biological morphometry. In: Stereological Methods. Academic Press, London, p 40-45

22. Hirose K, Osterby R, Nozawa M, Gundersen HJ 1982 Development of glomerular lesions in experimental long-term diabetes in the rat. Kidney Int 21:689-695

23. Wilson PD 2004 Polycystic kidney disease. N Engl J Med 350:151-164

24. Grantham JJ, Torres VE, Chapman AB, Guay-Woodford LM, Bae KT, King BF Jr, Wetzel LH, Baumgarten DA, Kenney PJ, Harris PC, Klahr S, Bennett WM, Hirschman GN, Meyers CM, Zhang X, Zhu F, Miller JP 2006 Volume progression in polycystic kidney disease. N Engl J Med 354:2122-2130

25. National Kidney Foundation 2002 K/DOQI clinical practice guidelines for chronic kidney disease: evaluation, classification, and stratification. Am J Kidney Dis 39:S1-S266

26. Levey AS, Greene T, Beck GJ, Caggiula AW, Kusek JW, Hunsicker LG, Klahr S 1999 Dietary protein restriction and the progression of chronic renal disease: what have all of the results of the MDRD study shown? Modification of Diet in Renal Disease Study group. J Am Soc Nephrol 10:2426-2439

27. De Keijzer MH, Provoost AP 1990 Effects of dietary protein on the progression of renal failure in the Fawn-Hooded rat. Nephron 55:203-209

28. Zeier M, Fehrenbach P, Geberth S, Mohring K, Waldherr R, Ritz E 1992 Renal histology in polycystic kidney disease with incipient and advanced renal failure. Kidney Int 42:1259-1265

29. Tanner GA, Tielker MA, Connors BA, Phillips CL, Tanner JA, Evan AP 2002 Atubular glomeruli in a rat model of polycystic kidney disease. Kidney Int 62:19471957

30. Korotkova M, Gabrielsson BG, Holmang A, Larsson BM, Hanson LA, Strandvik B 2005 Gender-related long-term effects in adult rats by perinatal dietary ratio of n-6/n-3 fatty acids. Am J Physiol Regul Integr Comp Physiol 288:R575-R579

31. Wiesenfeld PW, Babu US, Collins TF, Sprando R, O'Donnell MW, Flynn TJ, Black T, Olejnik N 2003 Flaxseed increased alpha-linolenic and eicosapentaenoic acid and decreased arachidonic acid in serum and tissues of rat dams and offspring. Food Chem Toxicol 41:841-855

32. Lefkowith JB, Klahr S 1996 Polyunsaturated fatty acids and renal disease. Proc Soc Exp Biol Med 213:13-23

33. Needleman P, Raz A, Minkes MS, Ferrendelli JA, Sprecher H 1979 Triene prostaglandins: prostacyclin and thromboxane biosynthesis and unique biological properties. Proc Natl Acad Sci USA 76:944-948

34. Grimminger F, Mayer K, Kramer HJ, Stevens J, Walmrath D, Seeger W 1993 Differential vasoconstrictor potencies of free fatty acids in the lung vasculature: 2-versus 3-series prostanoid generation. J Pharmacol Exp Ther 267:259-265

35. Wang L, Chen J, Thompson LU 2005 The inhibitory effect of flaxseed on the growth and metastasis of estrogen receptor negative human breast cancer xenograftsis attributed to both its lignan and oil components. Int J Cancer 116:793-798

36. Chen J, Wang L, Thompson LU 2006 Flaxseed and its components reduce metastasis after surgical excision of solid human breast tumor in nude mice. Cancer Lett 234:168-175

37. Fritsche KL, Johnston PV 1990 Effect of dietary alpha-linolenic acid on growth, metastasis, fatty acid profile and prostaglandin production of two murine mammary adenocarcinomas. J Nutr 120:1601-1609 